\title{
Elementos educativos en las unidades didácticas en la UNED, Costa Rica, 2017 y 2018: frecuencia de uso y posibilidades de mejora
}

\author{
Carmen Andrés Jiménez ${ }^{1}$ (D) \& Gloriana Anchetta Meza ${ }^{1}$
}

1. Universidad Estatal a Distancia (UNED), 474-2050 Mercedes de Montes de Oca, San José-Costa Rica; candres@uned.ac.cr, ganchetta@uned.ac.cr

\author{
Recibido 10-X-2019 • Corregido 17-II-2020 • Aceptado 25-II-2020 \\ DOI: https://doi.org/10.22458/ urj.v12i1.2913
}

\begin{abstract}
Education elements in textbooks at UNED, Costa Rica, 2017 and 2018: use frequency and improve possibilities". Introduction: The production of textbooks in the Costa Rican Distance University must use a series of elements needed for distance education. Objective: To identify the most important educational elements used by the editors. Methods: We used a questionnaire and an editor's focus group to identify the frequency for each element in 24 textbooks. Results: More than $90 \%$ of the didactic units contain: graphs or diagrams, charts, definitions, practical cases, didactic discourse, "attractions", examples and signs. Between 70 and $90 \%$ use: images, attention calls, logical sequence and inserted questions. Less than $70 \%$ use anecdotes, establishment of contrary points, humanization of the text, summaries, timelines and metaphors. Conclusions: While most of the recommended elements are used, there is room for improvement in the variety and subject adaptation of learning elements used in these textbooks.
\end{abstract}

Keywords: Distance education, mediation, foundational theories, quality education, curriculum design, focus groups.
RESUMEN. Introducción: La producción de libros de texto en la Universidad de Distancia de Costa Rica debe usar una serie de elementos necesarios para la educación a distancia. Objetivo: identificar los elementos educativos más importantes utilizados por los editores. Métodos: Utilizamos un cuestionario y un grupo focal con los editores para identificar la frecuencia de cada elemento en 24 libros de texto. Resultados: Más del $90 \%$ de las unidades didácticas contienen: gráficos o diagramas, cuadros, definiciones, casos prácticos, discurso didáctico, "atracciones", ejemplos y signos. Entre el 70 y el $90 \%$ usan: imágenes, llamadas de atención, secuencia lógica y preguntas insertadas. Menos del $70 \%$ usa anécdotas, establecimiento de puntos contrarios, humanización del texto, resúmenes, cronogramas y metáforas. Conclusiones: Si bien se utilizan la mayoría de los elementos recomendados, hay margen para mejorar la variedad y la adaptación de los elementos de aprendizaje utilizados en estos libros de texto.

Palaras clave: Educación a distancia, mediación, teorías del aprendizaje, calidad de la educación, grupos focales. 
Una unidad didáctica se define como el material didáctico escrito que contiene el desarrollo de los contenidos de una asignatura o de un curso en el modelo educativo a distancia, de acuerdo con los objetivos de aprendizaje, evaluación y metodología previamente establecida por el grupo de especialistas en el proceso de planificación curricular. Las unidades didácticas deben contar con la mediación pedagógica y las características de discurso y de calidad, en general, que favorezcan el aprendizaje a distancia.

Las unidades didácticas deben elaborarse bajo estrictos requerimientos de calidad y llenar requisitos mínimos para que los objetivos de aprendizaje se cumplan. La calidad de dichos materiales gira en torno a una serie de requerimientos relacionados con la educación a distancia. Este estudio se enfoca en tres de ellos: las estrategia didáctica o mediación pedagógica, contenidos y teorías de aprendizaje.

La mediación pedagógica se puede definir como un conjunto de actividades, recursos y medios didácticos que se utilizan para desarrollar el proceso educativo y el aprendizaje del estudiantado a fin de favorecer el aprendizaje significativo (Rojas, 2016). Se puede denominar también como estrategia didáctica, la cual, al escogerse de manera correcta, permite el logro de metas de enseñanza y aprendizaje (Hernández, 1993). Por esta razón, en un material escrito, el uso adecuado de estrategias que medien el aprendizaje del estudiantado posibilita la adquisición de conocimientos y la comprensión de los conceptos, aplicados a un área de desempeño y el análisis de resultados en forma crítica y analítica. Por otro lado, de acuerdo con un estudio realizado por Borgobello, Sartori y Sanjurjo (2018), encontraron que, respecto a las estrategias pedagógicas a utilizar, la preocupación fundamental entre el profesorado es la motivación del estudiantado.

Asimismo, en un estudio realizado por Teclehaimanot y Marshall (2016), encontraron ocho prácticas fundamentales para desarrollar contenido pedagógico en la educación superior a distancia: una correcta introducción, objetivos bien planteados, evaluación y medición, instrucciones, actividades para aprender e interactuar, uso de la tecnología, soporte para el alumnado y accesibilidad.

Por su parte, Hernández (1993) indica los elementos de una estrategia didáctica, entre los cuales se destacan: uso de organizadores, creación de espacios de aprendizaje como íconos, anécdotas o actividades de investigación, manejo de recursos idiomáticos en sus distintos aportes (compresión o ampliación del discurso escrito y la puntualización de conceptos clave). Así, se hace necesario considerar el aspecto pedagógico en un sistema de educación a distancia y en este caso en particular, en el diseño de los materiales didácticos escritos, para que así se logre una correcta oferta de materiales de calidad. García-Aretio (2015), termina diciendo que "el rendimiento de los estudiantes depende más de los diseños pedagógicos de cada acción formativa que de los recursos seleccionados para el aprendizaje" (p.128).

De acuerdo con García-Aretio (2015), un material que no contenga contenidos de calidad, metodológicamente enfocados, que no tenga personal docente calificado, con habilidades para el trabajo colaborativo, la interacción y conocimientos en educación a distancia y aspectos organizativos y de gestión que garanticen un buen funcionamiento de este sistema tan particular; no será de utilidad en una universidad a distancia. En este estudio se pretende discernir cómo integrar estos elementos en la producción de unidades didácticas para alcanzar los objetivos de aprendizaje y obtener un producto de calidad.

Para que los contenidos en una unidad didáctica sean de calidad, es básico considerar su desarrollo (García-Aretio, 2016) o el cuerpo del libro (Martínez de Sousa, 2007), el cual inicia con una introducción, en donde se explica la disposición general del capítulo, los antecedentes y cómo se abordarán los contenidos; seguidamente, se muestran los contenidos de acuerdo con lo que dictamina el diseño curricular de la asignatura, sin limitarse a un orden estricto, ya que como lo indica Hernández (1993), el medio impreso deberá tener su propia dinámica y organizar los 
contenidos en el orden en el que están en el diseño curricular puede hacer innecesariamente voluminosa y lineal la exposición de la temática y rutinarios la enseñanza y el aprendizaje para el estudiante (p.32); para finalmente concluir sobre lo estudiado y fomentar la autorregulación en el estudiantado.

García-Aretio (2015) señala que los contenidos que se utilizan en la educación a distancia deben ser: científicos, pedagógicos, económicos y estructurales. Un contenido científico, implica que sea fidedigno, actual, fiable y esencial. Un contenido pedagógico significa que debe ser planificado, adecuado, adaptable, integral, integrado, abierto, coherente, eficaz, transferible, significativo, interactivo y que permita la autoevaluación. Por otro lado, el que un contenido sea económico significa que sea eficiente, de manera que la inversión realizada en este sea rentable y estandarizado, para que pueda llegar por todos los medios si se trata de un material de carácter tecnológico. Finalmente, el contenido debe ser estructural; es decir, que tenga una secuencia lógica para comprender el contenido, debe ser enriquecido (que contenga imágenes, enlaces, cuadros) y atractivo, de manera que se aprovechen las posibilidades de presentación gráfica.

Finalmente, entre las teorías del aprendizaje que más aplican en las unidades didácticas se pueden citar cuatro: la teoría de la flexibilidad, la teoría sobre el aprendizaje contextualizado, la teoría de la cognición distribuida y la teoría del aprendizaje situado (Fallas \& Trejos, 2013). A continuación, se especifica cada una de ellas según Fallas y Trejos (2013):

- Según la teoría de la flexibilidad (de la psicología cognitiva), los estudiantes muchas veces aprenden en ambientes mal estructurados $y$, como consecuencia, se tienen resultados deficientes. Por el contrario, una estructuración lógica del curso dentro de la plataforma y de manera presencial, además de sus temas y actividades, con distintas perspectivas sobre un tema y con posibilidades de profundización es necesaria para permitir un aprendizaje lógico mentalmente, que permitan ir construyendo nuevos conocimientos.

- La teoría de Piaget y Bruner sobre el aprendizaje contextualizado de Piaget y Bruner, indica que las actividades relacionadas con la vida diaria del estudiante y con su experiencia previa permiten que se construyan nuevas ideas y conceptos. Por esto el aprendizaje debe estar estructurado de manera que se tomen como base los aspectos propios del estudiante, y de esta manera, guiarlo al alcance de la construcción de nuevos conocimientos.

- Por su parte, la teoría de la cognición distribuida (de la psicología cognitiva), en la educación se debe generar discusión y diálogo crítico, a través de las consignas de las actividades para encontrar principios que puedan aplicarse a distintos problemas. En efecto, de acuerdo con las necesidades de la sociedad del conocimiento, se necesita una persona que sea reflexiva, crítica y propositiva.

- Finalmente, la teoría del aprendizaje situado destaca la importancia de tutorías, trabajos colaborativos y herramientas cognitivas que se sirvan de tareas y actividades en contextos reales. Según esta teoría, se debe evitar un aprendizaje abstracto y descontextualizado, sino más bien uno que surja a partir del contexto y la cultura en la que se está inmerso, de manera que la construcción del conocimiento tenga una utilidad real en la vida profesional del estudiante.

Según la teoría cognitivista (Harasim, 2012, citado por Picciano, 2017), las funciones cerebrales tienen un papel fundamental en el aprendizaje y, por tanto, es necesario revisar la interacción del cerebro con el objeto de aprendizaje. En esta línea, cabe destacar la teoría instruccional de Gagné, que especifica nueve momentos para la elaboración de materiales instruccionales:

1. Captar la atención al utilizar medios relevantes para el estudiante.

2. Describir el objetivo por lograr. 
3. Estimular el aprendizaje previo y conectarlo al que se construirá de seguido.

4. Presentar los materiales por estudiar de forma clara y sencilla.

5. Brindar guías para el aprendizaje, de modo que el estudiante logre reflexionar y revisar constantemente su progreso.

6. Promover el protagonismo del estudiante a través de tareas interactivas y dinámicas.

7. Ofrecer retroalimentación inmediata, específica y constructiva.

8. Evaluar el desempeño del estudiante, mediante diferentes actividades de aprendizaje.

9. Promover la transferencia del aprendizaje, al incluir dinámicas de aprendizaje que acerquen los nuevos conocimientos a la realidad de los estudiantes (Picciano, 2017, p. 3).

González, Salcines y Ramírez (2018), diseñaron un grupo focal para determinar las características de dispositivos móviles y comunicación en familia. Ellos recomiendan tener una población heterogénea de participantes, para obtener la mayor cantidad de respuestas posibles a un mismo tema.

Onyumba, Wilson, Derrick y Mukherjee (2018) identificaron los cinco tipos de estrategias que se utilizan en la investigación: discusiones generales, discusiones detrás de un vidrio (dos grupos discuten sobre el mismo tema), uso de doble moderador (cada uno tiene un propósito y de esta forma se asegura se cubra todos los temas), el abogado del diablo (dos moderadores, cada uno con un punto de vista diferente para generar controversia), designar a algunos participantes como moderadores para generar respuestas más honestas y variadas y mini grupos focales.

El Programa de Producción de Material Didáctico Escrito (PROMADE) es una unidad académica que pertenece a la Dirección de Producción de Materiales (DPMD) de la Universidad Estatal a Distancia (UNED). La elaboración de materiales didácticos en PROMADE es un proceso delicado que responde a la necesidad de los diversos programas académicos que existen a nivel de Diplomado, Bachillerato y Licenciatura de la universidad.

Los materiales didácticos deben propiciar la autorregulación y la metacognición en el estudiantado. De acuerdo con García-Aretio (1997), los materiales impresos para la enseñanza a distancia deben motivar, transmitir eficazmente la información, aclarar dudas, mantener diálogo permanente con el alumno, orientarle, establecer las recomendaciones oportunas para conducir el trabajo, controlar y evaluar los aprendizajes" (p.2). Asimismo, dentro de las estrategias para potenciar la evaluación reguladora y auto reguladora dentro del Plan de Desarrollo Académico de la UNED (2012), que son necesarias para aprender a aprender; se menciona que el estudiante debe desarrollar una habilidad eficaz para regular el propio proceso de aprender, basado en pruebas sucesivas que permiten decidir y aplicar acciones correctivas para lograr el nivel de aprendizaje.

Es necesario que los equipos de PROMADE aseguren la presencia de los elementos anteriores en las unidades didácticas, para desarrollar en el estudiantado la capacidad de análisis que le permita darse cuenta de lo que ha aprendido, qué le falta por aprender y la manera de conseguirlo.

El objetivo de este estudio es determinar los atributos de calidad relacionados con la mediación pedagógica, los contenidos y las teorías de aprendizaje en la elaboración de unidades didácticas mediante un grupo focal. 


\section{MATERIALES Y MÉTODOS}

El tipo de investigación que se realizó fue de carácter mixto denominado Diseño anidado o incrustado concurrente de modelo dominante (DIAC), que colecta simultáneamente datos cuantitativos y cualitativos, pero uno de los métodos es el predominante (en este caso es el cuantitativo). El método con menor prioridad se anida al principal (Hernández-Sampieri, Fernández, \& Baptista (2010).

La experiencia fue basada en las vivencias de los profesionales en el campo de la edición de textos académicos de PROMADE. Forma parte de un proyecto de investigación que inició en el 2018 con el fin de determinar los lineamientos de calidad más importantes en la producción de unidades didácticas en PROMADE de la UNED de Costa Rica.

Se realizó en cuatro etapas, dos cualitativas y dos cuantitativas. En las cualitativas se elaboró y validó un cuestionario para llevar a cabo un grupo focal y en las cuantitativas se elaboró y validó un instrumento para evaluar la calidad de las unidades didácticas y se aplicó a una muestra de 24 unidades didácticas aplicación del instrumento para obtener resultados cuantitativos.

SECCIÓN CUALITATIVA: La sección cualitativa de esta investigación incluyó la elaboración y validación de un instrumento para aplicar un grupo focal.

Elaboración de lista de un cuestionario para el grupo focal con los lineamientos de calidad para productores académicos: Basado en una revisión bibliográfica, la normativa existente de la UNED y la experiencia de las investigadoras y otros productores de PROMADE, se elaboró un cuestionario que incluyó los lineamientos de calidad más importantes en la producción de unidades didácticas (Apéndice Digital 1). Las categorías que midió dicho instrumento fueron las siguientes: contenido de la unidad, mediación pedagógica y teorías de aprendizaje.

Asimismo, cada categoría poseía diferentes dimensiones según la recomendación de HernándezSampieri et al. (2010):

- Mediación pedagógica: discurso, formato y actividades de aprendizaje.

- Calidad en los contenidos: pedagógico, científico, económico y estructural.

- Teorías del aprendizaje: teoría del aprendizaje situado, teoría de la distribución cognitiva, teoría de la flexibilidad y teoría del aprendizaje contextualizado.

Con base en una investigación bibliográfica reciente, se indagaron las estrategias más adecuadas en el ámbito de la educación y producción de materiales didácticos para la elaboración de grupos focales y se determinó la que más se ajusta a cada variable trabajada.

Validación por parte de expertos de la lista de tópicos: Se realizó la validación de la lista de comprobación por partes de tres expertos de PROMADE, con el fin de determinar si la lista cumplía con su objetivo y si, con base en las preguntas se lograba el objetivo sobre lo que quiere indagar. Para esto, se utilizó el cuestionario del Apéndice Digital 2 y se tomaron en cuenta elementos como validez y criterio según Hernández-Sampieri et al. (2010), para investigaciones con enfoque cualitativo (Apéndice Digital 2).

Aplicación del grupo focal: una vez que se obtuvo el cuestionario validado por los productores académicos, se aplicó a ocho productores académicos mediante una sesión que duró dos horas y en la que se abordaron los tres temas de enfoque de este estudio: mediación pedagógica, calidad de los contenidos y teorías del aprendizaje. Para aplicar el grupo focal se escoge una muestra de expertos entre los productores académicos (que son veinte), de los cuales participaron ocho, lo cual 
es una muestra de expertos. Según Hernández (2016) la muestra de expertos es necesaria cuando se requiere la opinión de personas expertas en un tema (p. 387).

Análisis de resultados cualitativos del grupo focal: con base en los resultados obtenidos del grupo focal se determinaron categorías y dimensiones. Las categorías fueron: mediación pedagógica, calidad en los contenidos y teorías del aprendizaje. Cada categoría incluyó las siguientes dimensiones:

- Mediación pedagógica: metáfora pedagógica, resumen al final de cada capítulo, preguntas de aprendizaje, definiciones, gráficos, cuadros, llamadas de atención, líneas de tiempo, ilustraciones.

- Calidad del material: discurso didáctico, resaltar lo más relevante, ir de lo más sencillo a lo más complejo y ser atractivo.

- Teorías de aprendizaje: simulaciones de situaciones de la vida real, hilo conductor, secuencia lógica, uso de metáforas o ejemplos para comprender determinados conceptos.

SECCIÓN CUANTITATIVA: La sección cuantitativa de esta investigación incluyó la elaboración y validación del instrumento para evaluar 24 unidades didácticas elaboradas en PROMADE, entre el 2018 y 2019, a la luz de los resultados obtenidos en el grupo focal, sin esto significar que todas las estrategias de mediación pedagógica mencionadas en este ejercicio deben estar necesariamente presentes en las unidades didácticas, aunque su uso generalmente aporta mayor calidad al material didáctico.

Se realizó la selección de la muestra, la aplicación del instrumento y el análisis de los resultados arrojados en variables y dimensiones.

Elaboración de instrumento para evaluar las unidades didácticas: con base en los resultados obtenidos del grupo focal se elaboró un instrumento que incluyó 23 dimensiones relacionadas con las tres categorías de análisis. Se muestran en el Apéndice Digital 3.

Selección de muestra: Se utilizó una muestra diversa o de máxima variación (Hernández et al., 2010, p. 387) porque el objetivo es mostrar las distintas perspectivas y representar la complejidad del fenómeno. Si se seleccionara entre algunas unidades didácticas, se podría omitir información importante que pueda ser útil para el estudio o para PROMADE.

Aplicación del instrumento: se aplicó el instrumento a la muestra seleccionada de 24 unidades didácticas. Se tiene acceso a las versiones preliminares en PDF que se entregaron a OFIDIVE durante el 2017 y 2018.

Análisis de resultados cuantitativos: seguidamente, se incluyeron los resultados en una base de datos para clasificarlos, determinar los porcentajes de presencia en las 24 unidades didácticas analizadas y plasmarlos en gráficos que clasificaron las diferentes estrategias de mediación pedagógica evaluadas en tres variables:

- ¿Cuáles de las estrategias de mediación están presentes en más del $90 \%$ de las unidades didácticas?

- ¿Cuáles de las estrategias de mediación están presentes entre el $70 \%$ y el $90 \%$ de las unidades didácticas?

- ¿Cuáles de las estrategias de mediación están presentes en menos del 70\% de las unidades didácticas? 
Ética, conflicto de intereses y declaración de financiamiento: Las autoras declaran haber cumplido con todos los requisitos éticos y legales pertinentes, tanto durante el estudio como en el manuscrito; que no hay conflictos de interés de ningún tipo, y que todas las fuentes financieras se detallan plena y claramente en la sección de agradecimientos. Asimismo, están de acuerdo con la versión editada final del documento. El respectivo documento legal firmado se encuentra en los archivos de la revista.

\section{RESULTADOS}

Lista de tópicos para el grupo focal con los lineamientos de calidad para productores académicos: A partir de la investigación teórica realizada y de la evaluación de la validez del instrumento, se elaboró un cuestionario, cuyo contenido incluía las tres categorías que se trabajaron (mediación pedagógica, calidad en los contenidos y teorías del aprendizaje) (Apéndice Digital 1).

Validación por parte de expertos de la lista de tópicos: el cuestionario fue validado con el instrumento que se encuentra en el Apéndice Digital 2 y se obtuvieron diferentes respuestas para que se cumplieran los objetivos del ejercicio (Apéndice Digital 5).

Resultados del grupo focal: de seguido, se muestran los resultados obtenidos del grupo focal, que se realizó con ocho productores académicos y se dividió en tres categorías: mediación pedagógica, calidad de los contenidos y teorías del aprendizaje.

Respecto a la medicación pedagógica, en el grupo focal se afirmó que la mediación pedagógica es la esencia de los materiales didácticos de la UNED, por su naturaleza de estudio a distancia que, incluso, le funciona a otros usuarios externos. A los participantes del grupo focal se les consultó sobre las estrategias de mediación que utilizan con mayor frecuencia y las respuestas son las siguientes, que se han agrupado en tres categorías: discurso, formato y actividades de aprendizaje (Apéndice Digital 6). Ahora bien, en el grupo focal se habló del "uso y abuso" de estrategias didácticas; además, se debe dosificar la información y, en temas difíciles, redundar con diferentes estrategias pedagógicas.

Relacionado con la calidad en los contenidos, se enfocó en dos elementos: pedagógico y estructural. Las características que debe incluir una unidad didáctica para que los contenidos abordados sean de calidad, son las siguientes:

- Contenido pedagógico: Los materiales deben contener discurso didáctico que se asemeja al profesor en la clase presencial y se debe analizar qué es lo más relevante y la mejor manera de transmitirlo para así, lograr aprendizaje significativo.

- Contenido estructural: se debe abordar de lo más sencillo a lo más difícil y el autor debe conocer esto, pues no es solamente colaborar con los contenidos, sino que debe ayudar a estructurales, lo anterior debe analizarse desde el plan de la obra, se deben diferenciar con exactitud los materiales: unidades didácticas, guía de estudio, manual de laboratorio y debe ser atractivo y sencillo.

Finalmente, relacionado con las teorías de aprendizaje, se determinaron la manera en la que se pueden aplicar las diversas teorías del aprendizaje en las unidades didácticas y se discutió en el grupo focal (Apéndice Digital 7).

Aplicación del instrumento en las unidades didácticas: con base en los resultados que el grupo focal se realizó el análisis de las unidades didácticas. Cada unidad didáctica contiene una serie de elementos adicionales que no fueron contemplados en el grupo focal y que, por ende, no se incluyen 
en esta investigación. No significa lo anterior que no estén presentes dichos elementos en las unidades didácticas, que enriquecen aún más la calidad del material; por ejemplo: actividades de reflexión, códigos $Q R$, enlaces para audiovisuales, organizadores previos, pruebas de diagnóstico, estrategias para fomentar la autoevaluación, frases célebres y muchos otros.

Por otro lado, al aplicar el instrumento a las unidades didácticas, se encontraron los siguientes resultados, de acuerdo con las variables y las dimensiones previamente definidas y el que algunos elementos no están presentes en las unidades didácticas no aplica por las características de cada asignatura. También se encontró que el uso de los elementos es homogéneo en las unidades didácticas cuando está presente, dado que es parte del estilo de la persona editora académica (Apéndice Digital 8).

Presencia de las estrategias: Los elementos presentes en más del $90 \%$ de las unidades didácticas evaluadas son: casos prácticos (92\%), ejemplos (100\%), señalizadores (96\%), definiciones (100\%), gráficos (100\%), cuadros (92\%), discurso didáctico (100\%), ir de lo más sencillo a lo más complejo (100\%), ser atractivo (92\%) y secuencia lógica $(97 \%)$.

Los elementos presentes en menos del $90 \%$ de las unidades didácticas evaluadas son las preguntas de aprendizaje (80\%), las llamadas de atención (80\%) y las ilustraciones $(80 \%)$.

Los elementos presentes en menos del $70 \%$ de las unidades didácticas evaluadas son: metáfora pedagógica (20\%), anécdotas (40\%), puntos de vista contrarios (60\%), humanización del texto (33\%), resumen al final de cada capítulo (65\%) y líneas de tiempo (10\%).

Coincidencia con el grupo focal: Respecto a la coincidencia entre lo sugerido en el grupo focal y lo que se aplica en la realidad en las unidades didácticas, se concluye que existe un $58 \%$ de las unidades didácticas utilizan la mayor parte de las estrategias de mediación pedagógica, según lo propuesto en el grupo focal, mientras que el restante $42 \%$ no se encuentra presente en dicho material (Apéndice Digital 9).

\section{DISCUSIÓN}

Un estudio realizado por (González-Fernández, Salcines-Talledo, \& Ramírez-García, 2018). sobre la mediación pedagógica, encontró que el principal desafío en la educación superior de estos tiempos implica reflexionar sobre las necesidades de los alumnos, en especial las de aquellos que se les dificulta asistir en forma presencial a un centro educativo debido a factores de tiempo, distancia y economía. De esta manera, como lo demostró el grupo focal, la mediación pedagógica se vuelve fundamental en las unidades didácticas que se convierten muchas veces en el único medio que la comunidad estudiantil tiene para aprender.

Al respecto, en las unidades didácticas producidas en PROMADE en el periodo estudiado, se encontraron dichos elementos distribuidos según se observa en los resultados, de una manera heterogénea que aún demuestra la necesidad de estandarizar procedimientos, motivar a las personas productoras académicas a capacitarse en diferentes áreas y encontrar denominadores comunes que promuevan un aprendizaje significativo a través del material didáctico producido.

A continuación, se analiza la variable "mediación pedagógica". El uso de anécdotas se encontró en un $42,5 \%$ de las unidades didácticas evaluadas. No es aún la estrategia más común entre las unidades didácticas de PROMADE; sin embargo, su aplicación puede ser muy útil en la comprensión de un texto. Sánchez, López y Alfonso (2018) las utilizó en un estudio en Perú y obtuvo resultados positivos después de dicha aplicación porque el nivel de comprensión de lectura mejoró notablemente. 
Las estrategias como puntos de vista contrarios (encontrados en un 63\%), señalizadores (utilizados en un 96\%) y las llamadas de atención (encontradas en un 75\%), corresponden a estilos de control metacognitivo, utilizado por Fonseca, Migliardo, Simian, Olmos y León (2019) en un estudio donde el grupo control no recibió este tipo de estímulo y el grupo que sí lo recibió mostró mejoras estadísticamente significativas en la comprensión lectora. Se recomienda, por lo tanto, incluir estas estrategias pedagógicas de una manera más homogénea en las unidades didácticas, de forma que sean beneficiosas para el estudiantado.

La humanización del texto, encontrada en un $33 \%$ en las unidades didácticas, representa otro estilo de mediación pedagógica que facilita la comprensión de un texto. El tener información que vaya más allá de la mera mención del personaje histórico, permite contextualizar al estudiantado para que relacione la época o las características del personaje con la materia que se está desarrollando; tal como lo mencionan Larouche y Triviño (2019).

El uso de definiciones es completamente homogéneo dado que consiste en un requisito fundamental en todas las unidades didácticas de PROMADE. Su uso permite que el estudiantado se ubique en el tema que se está estudiando y tenga claro en qué consiste.

El uso de resumen se presenta en un $67 \%$ y se recomienda aumentar su uso, ya que corresponde a una estrategia alternativa de aprendizaje donde algunas personas pueden comprender mejor un tema, y complementan su aprendizaje, mientras que, al solamente leer la materia, puede que no lo logren en su totalidad.

Las líneas de tiempo se encontraron en un $12 \%$ de las unidades didácticas y es un recurso para el aprendizaje que se recomienda explorar más, así como lo hicieron los expertos en el grupo focal. En un estudio realizado por Paz (2019), en donde utilizó las líneas de tiempo como una estrategia innovadora de aprendizaje, un $70 \%$ del estudiantado mejoró su nivel de aprendizaje después de la aplicación.

El uso de cuadros, gráficos, mapas conceptuales y esquemas, utilizados en un $100 \%$, son herramientas de aprendizaje que permiten plasmar la información de una manera diferente, para que sea comprensible por toda la población estudiantil. Según Duarte, Lozano y Apolín (2019) estas estrategias promueven aprendizajes significativos y la motivación con los contenidos discursivos.

Seguidamente, se analiza la variable "calidad en los contenidos". El diseño gráfico se considera un factor de calidad en las unidades didácticas y el uso de ilustraciones, cuya incidencia fue del $83 \%$, es un elemento que se puede mejorar mediante capacitación por parte de expertos, para potencializar su uso en estos materiales. Al respecto, un estudio de Guerrero y Villegas (2019), enfatizó en la importancia del diseño gráfico para el aprendizaje, pues permite la comprensión de significados, interpretación y ejercicio de acciones (párr. 2).

Se demostró que las unidades didácticas de PROMADE, en su totalidad, están escritas de lo más sencillo a lo más complejo, lo cual potencia el desarrollo de habilidades cognitivas y competencias en el estudiantado. En un estudio realizado por Ferrales y Pérez (2020), sobre la metodología por utilizar cuando se resuelven problemas profesionales, se definió que los contenidos deben secuenciarse de lo simple a lo complejo, de de lo conocido a lo desconocido, de lo abstracto a lo concreto, teniendo en consideración la lógica de la profesión y su relación con el proceso docente - educativo (p. 83).

En el grupo focal, en relación con esta variable, los participantes coincidieron en el que el contenido pedagógico debe apuntar hacia un aprendizaje significativo. Esto concuerda con la investigación realizada por Vicedo (2015) sobre la educación médica superior, afirma que la transmisión del conocimiento científico no es suficiente para lograr el aprendizaje de los estudiantes, sino que para expresar de manera pedagógica un conocimiento de hechos o conceptos, se requiere entender las estructuras del tema. Lo anterior se logra mediante el uso del discurso 
didáctico, que se encuentran en el 100\% de las unidades didácticas evaluadas, lo que demuestra la calidad de las unidades didácticas en este sentido.

Finalmente, respecto a la variable "teorías de aprendizaje", el hecho de que en el $90 \%$ de las unidades didácticas evaluadas incluye ejemplos, casos prácticos y simulación de casos de la vida real muestra un nivel de calidad alto en las unidades didácticas. Investigaciones similares recomiendan esta dimensión como el estudio realizado por López, Chavira y Fuentes (2016), donde los estudiantes adultos que participan del modelo de estudio a distancia prefieren espacios que les permitan solucionar los problemas de manera práctica, analizar las situaciones de forma crítica y comparar sus experiencias con los nuevos contenidos. La dimensión anterior, conocida como organizador previo, no fue uno de los elementos que se tocaron en el grupo focal; sin embargo, es un pilar fundamental en las unidades didácticas y se recomienda indagar su presencia en otras investigaciones.

El uso de casos prácticos y relacionados con la realidad está también relacionado con la teoría del aprendizaje situado, mediante la cual, el estudiantado aprende de acuerdo con el contexto por medio de ejemplos reales, estrategia recomendada en el grupo focal. Dicho resultado es similar al estudio de Serrano (2019) sobre un modelo basado en casos de la vida real para preparar profesionales mediante cápsulas de autoaprendizaje y una aplicación móvil y encontró que dichas tácticas motivan al estudiantado a adoptar un rol participativo y cumplir con los objetivos propuestos. Asimismo, D’Agostino, Meza y Cruz (2003) recomiendan la relación con la realidad nacional y el ámbito laboral y profesional.

De acuerdo con Meza (2013), los contenidos de un programa a distancia deben estar organizados de modo que sean coherentes entre sí, lógicos y logren el objetivo propuesto. De otro modo, si no hay una estructuración de los contenidos que se abordan en el material, generarán desmotivación del estudiante y eventualmente la deserción. Según el grupo focal, se vuelve necesario el papel del plan de la obra, en el que se ordenan los temas por abordar, de acuerdo con diseño curricular y se aprueba por todo el equipo de producción; lo cual, concuerda con la teoría de la flexibilidad. Tal estrategia estuvo presente en más del $100 \%$ de las unidades didácticas y demuestra la calidad y profesionalismo de los materiales realizados en PROMADE.

En el grupo focal, respecto a las teorías del aprendizaje en la educación a distancia, se rescataron las características de las teorías cognitivistas y constructivistas. El uso de la teoría de distribución cognitiva, según los expertos consultados, implica que haya un hilo narrativo dentro de la unidad didáctica y un discurso de preguntas y respuestas que permitan una asimilación de conceptos correcta, así como su aplicación y análisis. En el análisis de las unidades didácticas, se encontró que, en las preguntas intercaladas de aprendizaje, la incidencia fue del $87 \%$, el cual puede aumentarse para mejorar la calidad de tales materiales.

Finalmente, con respecto a la teoría del aprendizaje contextualizado, los participantes del grupo focal acordaron que el uso de metáforas en el discurso académico es útil para afianzar conocimientos. Dicho resultado concuerda con el obtenido por Upegui (2009), quien utilizó esta estrategia en el discurso académico de artículos académicos y concluyó que resulta un recurso cognitivo que fortalece los objetivos de comunicación y la comprensión textual, además de expresar valores específicos de una cultura. Al haberse utilizado solamente en un $25 \%$ de los casos, se recomienda fortalecer su uso mediante capacitaciones donde se comprenda mejor su aplicación y los beneficios que provee al estudiantado.

Se recomienda retomar la figura del plan global dentro de PROMADE, para estandarizar su uso entre las personas productoras académicas y así, obtener mejores resultados. Además, se recomienda fomentar el uso de la secuencia lógica y las preguntas intercaladas para mejorar la calidad de las unidades didácticas nuevas. Finalmente, se recomienda tomar siempre el público 
meta, evitar el uso y abuso de estrategias de mediación pedagógica en las unidades didácticas y utilizar ejemplos que tengan relación con situaciones de la vida real.

\section{REFERENCIAS}

Borgobello, A., Sartori, M., \& Sanjurjo, L. O. (2018). Concepciones de docentes sobre los pedagógicas. Educación y Educadores, 21(1), 27-48.

estudiantes y sus prácticas

Fallas, I y Trejos, I. (2013). Educación en la sociedad de la información y el conocimiento. San José, Costa Rica: EUNED.

Ferrales, A. L. T., \& Pérez, P. A. Z. (2020). Metodología para la solución de problemas profesionales desde la didáctica de la formación de profesionales. REFCaIE: Revista Electrónica Formación y Calidad Educativa. ISSN 1390-9010, 7(3), 77-90.

Fonseca, L., Migliardo, G., Simian, M., Olmos, R., \& León, J. A. (2019). Estrategias para Mejorar la Comprensión Lectora: Impacto de un Programa de Intervención en Español. Educational Psychology, 25(2), 91-99.

García-Aretio, L. (1997). Una propuesta de estructura de Unidad didáctica y de guía didáctica. Publicado en García-Aretio, L. (Ed). El material impreso en la enseñanza a distancia. Madrid: UNED, 165-191.

García-Aretio, L. (2015). Bases, mediaciones y futuro de la educación a distancia en la sociedad digital. Madrid: Editorial Síntesis.

González-Fernández, N., Salcines-Talledo, I., \& Ramírez-García, A. (2018). Dispositivos móviles-smartphones \& tablets-y comunicación en familia diseño de un focus group. Prisma Social, (20), 21-39.

Guerrero, N. \& Villegas, A. (2019). La evaluación en el diseño gráfico como un factor de calidad para la funcionalidad de las soluciones gráficas. Revista Iberoamericana de Producción Académica y Gestión Educativa, 6(11).

Larouche, M \& Triviño, L. (2019). El uso escolar del patrimonio cultural en las ciencias sociales y la contribución de lo digital: algunas líneas de investigación en Quebec.

López, F., Chavira, H. y Fuentes, M. (2016). Condiciones del aprendizaje en los sistemas de educación a distancia. Culcyt / Educación 13 (1). Recuperado de

http://web.a.ebscohost.com.cidreb.uned.ac.cr/ehost/pdfviewer/pdfviewer?vid=3\&sid=d779771c-887e-4c0ca6b5-8486ba7f3aa7\%40sessionmgr4009.

Martínez de Sousa, J. (2007). Manual de estilo de la lengua española (MELE 3). Gijón: Trea, S.L.

Meza, J. (2013). Modelo pedagógico para proyectos de formación virtual. GIZ. Ministerio Federal de Cooperación Económica y Desarrollo. Recuperado de https://gc21.giz.de/ibt/var/app/wp342P/1522/wpcontent/uploads/2013/02/Ebook-final.pdf

D’Agostino, G., Meza, J., y Cruz, A. (2003). Elementos y características del material impreso que favorecen la formación y el aprendizaje a distancia en la UNED. San José: EUNED.

Picciano, A. (2017). Theories and frameworks for online education: Seeking an integrated model. Online Learning, 21(3), 166-190. doi: 10.24059/olj.v21i3.1225

O Nyumba, T., Wilson, K., Derrick, C. J., \& Mukherjee, N. (2018). The use of focus group discussion methodology: Insights from two decades of application in conservation. Methods in Ecology and Evolution, 9(1), 20-32.

Rojas, A. (2016). Planeamiento del proceso de enseñanza-aprendizaje. San José: EUNED.

Serrano, E.(2019). Modelo de Aprendizaje Basado en Casos y Problemas mediante la Integración de Capsulas de Autoaprendizaje y Aplicaciones Móviles Instrumentales en el la Formación del Personal de Salud (CAPPS). Universitas Tarraconensis. Revista de Ciències de l'Educació, 1(2), 43-51. 
Teclehaimanot, B. \& Marshall, H. (2016). Faculty Members' Best Practice Standards in the Design of Higher Education Online Courses. En $30^{\text {th }}$ Annual Proceedings. Volumes 1 \& 2.

Upegui, A. (2009). El uso de metáforas en tres artículos académicos de educación virtual. Lingüística y literatura, (53), 159179.

Vicedo, A. (2015). ¿Quién debe enseñar qué cosa en la educación superior médica? El papel del profesor y el conocimiento pedagógico del contenido. Educación cubana de médica superior 29 (3). Recuperado de http://web.b.ebscohost.com.cidreb.uned.ac.cr/ehost/pdfviewer/pdfviewer?vid=8\&sid=64ae0e55-d362-4163bd99-58d355d01719\%40pdc-v-sessmgr03 